\title{
Analysis of the effect of a series of back twist blade configurations for an active pitch-to-stall floating offshore wind turbine
}

\author{
Dawn Ward ${ }^{1}$, Maurizio Collu², Joy Sumner ${ }^{3}$ \\ ${ }^{1}$ Centre for Renewable Energy Systems, Cranfield University, Cranfield, Bedfordshire, MK43 \\ 0AL, United Kingdom. Email: dawn.ward@cranfield.ac.uk \\ ${ }^{2}$ Department of Naval Architecture, Ocean \& Marine Engineering, University of Strathclyde, \\ Henry Dyer Building, 100 Montrose Street, Glasgow, G4 OLZ, United Kingdom. \\ Email: maurizio.collu@strath.ac.uk \\ ${ }^{3}$ Centre for Thermal Energy Systems and Materials, Cranfield University, Cranfield, \\ Bedfordshire, MK43 0AL, United Kingdom. Email: j.sumner@cranfield.ac.uk
}

Corresponding author: dawn.ward@cranfield.ac.uk Tel: 01234619447

\begin{abstract}
For a turbine mounted on a floating platform, extreme induced loads can be increased by up to 1.6 times those experience by a turbine situated on a fixed base. If these loads cannot be reduced, towers must be strengthened which will result in increased costs and weight. These tower loads would be additionally exasperated for a pitch-to-feather controlled turbine by a phenomenon generally referred to as 'negative damping', if it were not avoided. Preventing negative damping from occurring on a pitch-to-feather controlled floating platform, negatively affects rotor speed control and regulated power performance. However, minimising the blade bending moment response can result in a reduction in the tower fore-aft moment response, which can increase the tower life. A variable speed, variable pitch-to-stall floating semisubmersible wind turbine, which does not suffer from the negative damping and hence provides a more regulated power output, is presented. This incorporates a back twist blade profile such that the blade twist, starting at the root, initially twists towards stall and, at some pre-determined 'initiation' point, changes direction to twist back towards feather until the tip. Wind frequency weighting was applied to the tower axial fatigue life trends of different blade profiles and a preferred blade back twist profile was identified. This had a back twist angle of $-3^{\circ}$ and started at $87.5 \%$ along the blade length and achieved a $5.1 \%$ increase in the tower fatigue life.
\end{abstract}

Keywords: floating offshore wind turbine (FOWT); pitch-to-stall; blade back twist; tower axial fatigue life

\section{Introduction}

Increasing the proportion of our energy that comes from renewable sources is imperative. Across the globe, extreme environmental events are increasing driven by indisputable anthropogenic contributions to climate change [1]. Unfortunately, this disproportionately effects the world's poorest people, which has likely contributed to the lack of adequate speedy action on the part of the world's economic leaders. If greenhouse gas emissions continue 
to rise, then it is predicted that the planet will experience a $1.5^{\circ} \mathrm{C}$ human-orchestrated global warming impact by 2030 [2]. This will cause wide spread issues with food, water security, flooding, droughts and storm conditions, which will shadow the devastating impact from global warming, that has already been seen in recent years.

Some countries are moving towards renewable energy faster than others and, within Europe, over $11 \%$ of energy was derived from wind power in 2018 , with an increasing proportion of nearly $10 \%$ of that wind energy coming from offshore farms [3]. Floating offshore wind turbines (FOWTs), which permit the use of a wider range of offshore sites, thus increasing global offshore wind potential, are still in the developmental stage. However, worldwide predictions are for a 5\% contribution of the global offshore wind energy market by 2030 [4].

The loads experienced by turbines situated on floating platforms, rather than a fixed base, are often increased, varying in both magnitude and origin, depending upon the platform design as well as on environmental conditions. Extreme induced tower base bending moments of a FOWT, have been shown to increase by up to 1.6 times those experienced by the same turbine with a fixed base [5]. If these loads cannot be reduced, towers will need additional strengthening, resulting in increased costs and weight.

As the frequencies of the floating platform's motions (surge, sway, roll and pitch) are generally one or more orders of magnitudes lower than for fixed turbines, when the turbine pitches-to-feather to control the rotor speed above rated wind speeds, a reduced performance is seen. This results in the rotor speed regulation not achieving a smooth response, and hence, negatively effecting the generated power output [6]. This performance drop is caused by the need to set the pitch control bandwidth at a lower frequency to avoid the first resonance modes of the turbine tower and thus avoid the negative damping, which can occur due to the continuous reduction in rotor thrust seen when pitching-to-feather. If unchecked, negative damping can create instability in the tower fore-aft response, as well as oscillations and hence increased tower fatigue. One solution to improve the regulation of the rotor speed and power generation of floating turbines could be to utilise turbines that still provide variable-speed, variablepitch control but by, instead, pitching towards stall. This is because, as found by Larsen and Hanson [6], this control method is not susceptible to 'negative damping' issues. The avoidance of negative thrust issues occurs since, as the wind velocity increases (in the above rated wind speed range), the rotor thrust increases for a stall controlled turbine, whereas for a feather controlled turbine the thrust diminishes, creating a negative derivative with respect to the wind speed [7]. Hence, active pitch-to-stall control enables a higher control frequency setting to be employed within the blade pitch controller, which improves both rotor speed regulation and power quality. However, pitching to stall often results in higher blade loads as well as deflection [8], although the peaks seen in 
passive stall blades are less likely to occur on an actively pitching-to-stall turbine. Also, thrust and torque are more stable as the pitch angle variations during operation in Region 3 are small for stall control compared to feather [9].

There have been only two studies (unrelated to this research), which have looked at the simulated responses of a floating turbine with active variable speed, variable pitch-to-stall (VSVP-S) control. However, these both utilized blades predominantly designed for feather control [6] [10]. Also, although reducing the tower response is often highlighted as a main design criteria for floating turbines due to the increased loads, research has generally focused on platform pitch damping [10] [11] [12]. However, reducing the blade bending moment response can also dampen the tower response, as has been observed with bend-twist coupled blades. These blades can be created by changes to the curved blade geometry in terms of twist, pre-bending, or the deflection that occurs under loads [13]. Twist, pre-bending or sweep are generally designed into the blade and implemented during manufacture and, under load, additional twist and bend will also be experienced. The blade can also be designed such that a deflection under load creates a reduction in the blade bending moment as the wind speed increases. Blade twist is standardly employed on large blades [13] [14] [15] and has been shown to reduce blade vibrations and fatigue as well as tower fore-aft frequency response on the $10 \mathrm{MW}$ DTU (The Technical University of Denmark) turbine, when pitching-to-feather [13]. Reversal of the direction of the blade twist profile at some point along the length, referred to as 'back twist' throughout the remainder of this study, has also been employed for fixed-stall blades. Thus, the blade twist, starting at the root, initially twists towards stall and, at some pre-determined 'initiation' point, changes direction to twist back towards feather until the tip. This helps to keep the airflow attached to the blade nearer the tip at higher wind speeds, which increases the power production for that section of the blade [14]. Generally the back twists explored have been of only a couple of degrees but have varied in initiation point from approximately 5 to $70 \%$ along the blade radius, measured from the tip [13] [14]. The design criteria of blade twist often focuses on maximising the turbine's power, as such, changing the setting of the constant pitch angle below rated wind speed has also been employed to optimize the energy output [9]. This work builds on the research presented on a VSVP-S FOWT by Ward et al [7], which investigated the effect on tower axial fatigue of a blade back twist that started at a distance of $75 \%$ along the blade radius, as measured from the root. For the semisubmersible platform analysed, an increase in the tower axial fatigue life of over $20 \%$ was achieved when operating in turbulent mean winds of $13 \mathrm{mps}$. These results highlighted the requirement for a further systematic investigation into the benefits in terms of tower life extension, due to changes in the back-twist magnitude or on the role of its initiation point on a VSVP-S controlled FOWT. 
Ostachowicz et al [16] state that, as the twist distribution of a coupled blade is dependent upon the wind speed, the blade cannot therefore be designed with a set tip-speed-ratio. Two methods to determine the non-optimal blade twist are proposed by [16]. The blade twist of a coupled blade is classed as non-optimal since fatigue is often reduced, but this may be at the expense of a reduction in the power generated. The first method is an iterative procedure that starts with the ideal blade twist for a specific tip-speed ratio, and which is then compared to the pre-twist required to achieve this at a specific wind speed. Iterative procedures used to optimise both blade twist and chord of a site specific passive stall blade, have however experienced difficulty at the early stages of the process, as the required angle of attack was outside the operational range [17]. The second method suggested was to optimise the blade twist at a specific wind profile. However, the authors that proposed this approach believed it has not yet been explored [16]. Currently there are no large, VSVP-S blade designs in use on floating platforms, partly because of a lack of understanding of a stalled blades aerodynamic behaviour [9]. Thus the iterative procedure would be more complex to apply than optimisation at a specific wind speed, as assessing the suitability of a specific twist is not a question of optimising an already commissioned blade design with an ideal twist for a specified tip-speed ratio.

This paper therefore aims to explore the effects of both the back twist's initiation point and its twist angle under a specific wind profile. The main desired outcome of presenting a preferred blade back twist for a specific site, is to reduce the tower fore-aft moment response and hence increase the tower fatigue life of a floating offshore semisubmersible wind turbine without overly adversely affecting the energy generated. The effect on both tower fatigue and energy generation of the constant pitch angle below rated wind speed setting will also be examined. A comprehensive optimisation would be beyond the scope of this work, since it should include a more advanced aero-elastic model of the blades and tower.

\section{Simulation Model}

\subsection{Dynamics model approach}

The aero-hydro-servo-elastic simulation models used in this study are based on the open source semi-submersible floating platform coupled to a 3 bladed 5 MW horizontal axis wind turbines (HAWT) and moored with 3 catenary mooring lines modelled utilizing MoorDyn. The mooring system dynamics are predicted assuming that each of the 3 mooring lines are a lumped mass and also accounts for the effects due to the buoyancy forces, weight, damping and axial forces as well as the hydrodynamic forces that have been derived utilizing Morison's equation. More details of the mooring system design can be found in the international OC4 Phase II study [18] [19] 20]. 
This OC4 Phase II study compared several offshore simulation code packages. The open source aero-hydro-servoelastic fully coupled time domain simulation code package, FAST v8 [21], was employed to generate a range of platform and turbine responses in different environmental settings. FAST v8 can predict aerodynamic loading as well as rotor and wake effects on the blades and, for a floating structure, the aerodynamic models are linked to hydrodynamic, dynamic structural and control models, allowing fully-coupled nonlinear aero-hydro-servo-elastic simulations within the time domain to be performed. FAST utilizers the Leishman-Beddoes dynamic stall model executed through AeroDyn. The potential flow theory hydrodynamic approach was implement in this study and utilized the frequency domain code WAMIT to derive the $1^{\text {st }}$ and $2^{\text {nd }}$ order hydrodynamic coefficients [18] [19] [20]. Identifying errors within aero-hydro-servo-elastic simulation codes and/or models can be challenging due to the complex nature of the loads under analysis, however the OC4 Phase II study simulation results from the different simulation code packages are all open source, which allowed for model and initial response validation. More details of the capabilities of the FAST simulation code can be found in the documentation by Jonkman and Buhl [21].

\subsection{Methodology}

The $1 / 50^{\text {th }}$ Froude-scaled experimental model as used as part of the US DeepCWind project [22], is the foundation of the simulation model of the semi-submersible floating platform, and is connected to the seabed by three catenary mooring lines. The tower of the $5 \mathrm{MW}$ NREL (National Renewable Energy Laboratory) turbine was amended as part of the OC4 phase II study [18], such that the mode shapes of the tower were altered to make them suitable for the additional flexibility required when coupled to this type of floating platform. Hence, the connection between the tower base and the platform is represented as a cantilever beam rather than the rigid connection used for a fixed base turbine. The turbine has a rotor diameter of $126 \mathrm{~m}$ and a hub height of $90 \mathrm{~m}$ above sea water level (SWL). The rated rotor speed is $12.1 \mathrm{rpm}$, which is achieved at a wind speed of $11.4 \mathrm{mps}$ and it is a variable speed, variable pitch-to-feather wind turbine. Further details on all aspects of the simulation models can be found in Robertson et al [18] and Jonkman et al [20].

There are three main models defined in the present work.

1. A conventional 'Feather Base Model' against which all other models are compared and which pitchesto-feather utilizing a constant-torque pitch controller with gain scheduling that avoids negative damping, as defined within the OC4 Phase II study [18]. 
2. A VSVP-S wind turbine, which has all the same simulation model characteristics as the 'Feather Base Model', with the exception that the lift coefficients of the blade aerofoils, which have been altered so that a smoother transition occurs with changes in the angle-of attack, as suggested and provided by [10]. This model, which pitches-to-stall is generally referred to as the 'Stall Test Model'.

3. A 'Stall Back Twist Model', which pitches-to-stall and which incorporates the changes made to the blade lift coefficients of the 'Stall Test Model' and further imposes a linear back twist towards feather. Thus, the blade initially starts to twist at the root, first towards stall and then at some pre-determined point changes direction to twist back towards feather until the tip, whereupon the specified back twist angle is reached. The basic 'Stall Back Twist Model' is further divided into a series of 12 models, each with a unique equispaced combination of back twist angle and initiation point as outlined in Table 1.

Initially, short 600s simulations were carried out at twist distances (at equally spaced intervals) for most of the blade length. However, at higher initiation points from the tip more blade vibrations were observed along with other simulation problems. Hence, the twists initiated have been confined to the last $25 \%$ of the blade (closer to the tip). Further details on the rotor thrust, power curves and pitch angles for the Feather Base Model and Stall Test Model, as derived through steady state analysis over the full range of operational wind speeds, are provided by Ward et al [7].

Table 1. Model parameters

\begin{tabular}{|c|c|c|c|c|c|c|}
\hline \multirow[t]{2}{*}{ Test ID } & \multirow{2}{*}{$\begin{array}{c}\text { Active variable } \\
\text { pitch blade } \\
\text { control }\end{array}$} & \multicolumn{2}{|c|}{$\begin{array}{l}\text { Twist starting distance } \\
\text { from bade root }\end{array}$} & \multirow{2}{*}{$\begin{array}{l}\text { Twist angle } \\
\text { increase at } \\
\text { the tip }\left(^{\circ}\right)\end{array}$} & \multirow{2}{*}{$\begin{array}{c}\text { Proportional } \\
\text { gain at } 18 \mathrm{mps}, \\
\mathrm{K}_{\mathrm{p}}(\mathrm{s})\end{array}$} & \multirow{2}{*}{$\begin{array}{c}\text { Integral } \\
\text { gain at } 18 \mathrm{mps} \text {, } \\
\mathrm{K}_{\mathrm{i}} \\
\end{array}$} \\
\hline & & $(\%)$ & $(\mathrm{m})$ & & & \\
\hline Feather Base Model & Feather & 0 & 0 & 0 & \multicolumn{2}{|c|}{ Inbuilt gain scheduler } \\
\hline Stall Test Model & Stall & 0 & 0 & 0 & -0.865 & -0.371 \\
\hline Stall Back Twist Model 1 & Stall & 75.0 & 46.1 & -3 & -0.917 & -0.393 \\
\hline Stall Back Twist Model 2 & Stall & 87.5 & 53.8 & -3 & -0.895 & -0.383 \\
\hline Stall Back Twist Model 3 & Stall & 92.8 & 57.0 & -3 & -0.880 & -0.377 \\
\hline Stall Back Twist Model 4 & Stall & 75.0 & 46.1 & -6 & -1.105 & -0.474 \\
\hline Stall Back Twist Model 5 & Stall & 87.5 & 53.8 & -6 & -0.981 & -0.421 \\
\hline Stall Back Twist Model 6 & Stall & 92.8 & 57.0 & -6 & -0.934 & -0.400 \\
\hline Stall Back Twist Model 7 & Stall & 75.0 & 46.1 & -9 & -1.414 & -0.606 \\
\hline Stall Back Twist Model 8 & Stall & 87.5 & 53.8 & -9 & -1.104 & -0.473 \\
\hline Stall Back Twist Model 9 & Stall & 92.8 & 57.0 & -9 & -1.000 & -0.429 \\
\hline Stall Back Twist Model 10 & Stall & 75.0 & 46.1 & -12 & -1.706 & -0.731 \\
\hline Stall Back Twist Model 11 & Stall & 87.5 & 53.8 & -12 & -1.216 & -0.521 \\
\hline Stall Back Twist Model 12 & Stall & 92.8 & 57.0 & -12 & -1.016 & -0.435 \\
\hline
\end{tabular}




\subsection{Control strategy approach}

To pitch the turbine blades towards stall to control rotor speed by keeping the rotor torque constant when wind speeds are above the rated speed of $11.4 \mathrm{mps}$, a feedback Proportional-Integral (PI) controller was built in Simulink and coupled to the FAST code as depicted in Fig 1. The controller gain settings are constant throughout the wind speed range with values derived at $18 \mathrm{mps}$ steady winds for each model. The controller also incorporated a pitch rate limiter set to the industrial norm of $+/-8 \% \mathrm{~s}$ [23] and an anti-windup filter [24]. At wind speeds below rated, the controller did not change the pitch angle for either the feather or stall designs. More details of this controller and the process to obtain the gain settings can be found in Ward et al [7].

The proportional and integral gains were unique to each stall model and were derived using a single degree of freedom (DOF) model of the angular shaft rotation as detailed by Larsen and Hanson [6], and Jonkman et al [20]. The derivative gain, $\mathrm{K}_{\mathrm{D}}$, has been set to zero as proposed by Hansen et al [25]. A constant gain derived at steady winds of $18 \mathrm{mps}$ was chosen for each model that was actively pitching-to-stall, as the pitch angle becomes doublevalued, such that the same blade pitch angle is seen at different wind speeds within Region 3 which would make the implementation of a gain scheduler more complex [20].

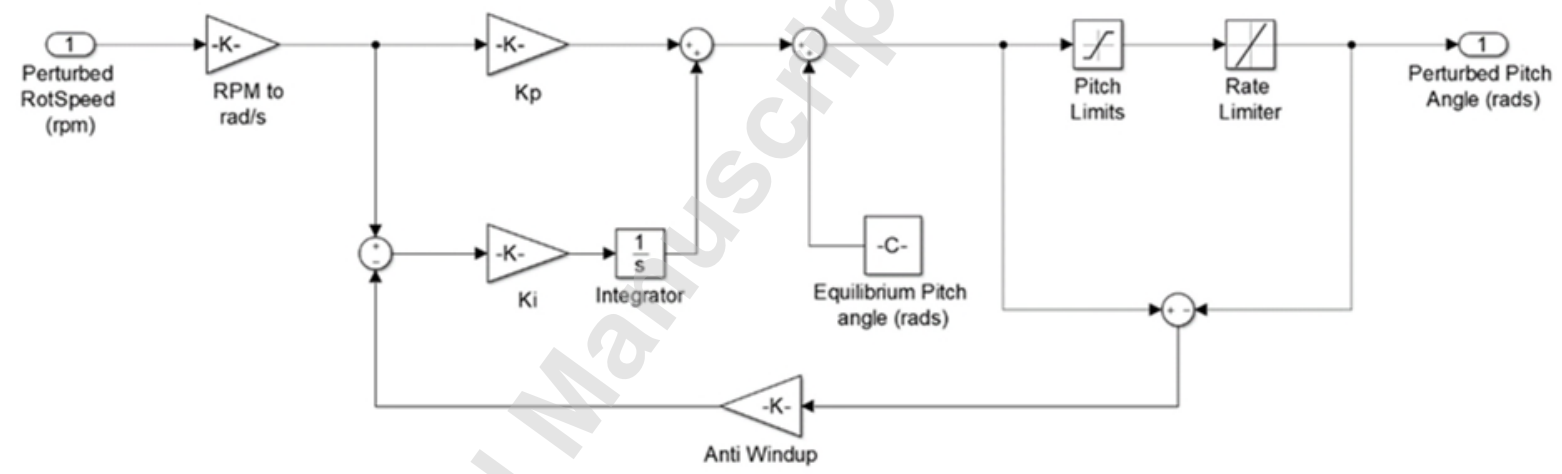

Figure 1. Pitch-to-stall feedback proportional-integral controller

\section{Test conditions}

To better understand the trends and effects of both back twist magnitude (BTM) and back twist initiation point (BTIP), a selection of relevant responses at 3 separate mean turbulent wind speeds were analysed. The specific environmental conditions were for a site based in the North Sea approximately 100 miles north-east of Aberdeen [26]. The sea depth for the site was unmeasured and so has been estimated at $200 \mathrm{~m}$. Table 2 , shows the conditions applicable for a turbine with a hub height of $90 \mathrm{~m}$ above SWL, as derived from the data accumulated by Geos [26], with the highlighted pathways applicable to the 3 mean stochastic turbulent wind speeds analysed. The mean 
turbulent wind speed of $8 \mathrm{mps}$ was chosen to represent the below rated wind speed conditions, as the $1^{\text {st }}$ tower fore-aft bending natural frequency occurs when the rotor speed is estimated to be approximately $9.2 \mathrm{rpm}$. The second mean turbulent wind speed chosen was $13 \mathrm{mps}$, as this is representative of the wind speed region around the rated wind speed of $11.4 \mathrm{mps}$. Lastly, a mean turbulent wind of $18 \mathrm{mps}$ represents the upper operational range of the turbine, where the wind speed reached, due to turbulence, an upper limit approaching the 25 mps cut off wind speed, at which the turbine would be expected to close down to prevent damage occurring.

Table 2. Average annual environmental conditions at grid point 59.300N 0.475E

\begin{tabular}{|c|c|c|c|c|c|c|c|c|}
\hline $\begin{array}{c}\text { Hub height } \\
\text { wind speed } \\
\text { (mps) }\end{array}$ & $\begin{array}{c}\text { Hub } \\
\text { height } \\
(\mathrm{m})\end{array}$ & $\begin{array}{c}\text { Hellman } \\
\text { exponent } \\
\text { (unstable air) }\end{array}$ & $\begin{array}{l}\text { Wind } \\
\text { height } \\
(\mathrm{m})\end{array}$ & $\begin{array}{l}\text { Wind } \\
\text { at site } \\
(\mathrm{mps})\end{array}$ & $\begin{array}{c}\text { Significant } \\
\text { Wave Height } \\
\text { Hs }(\mathrm{m})\end{array}$ & $\begin{array}{c}\text { Spectral } \\
\text { Peak Period } \\
\mathrm{T}_{\mathrm{p}}(\mathrm{s}) \\
\end{array}$ & $\begin{array}{c}\text { Windspeed } \\
\text { Range at } \\
\text { site (mps) }\end{array}$ & \begin{tabular}{|c}
$\%$ of total \\
operating \\
time
\end{tabular} \\
\hline & & & & & & & $0.3-1.6$ & \\
\hline & & & & & & & $1.6-3.4$ & \\
\hline 3 & 90 & 0.06 & 10 & 2.63 & \multirow{3}{*}{0.5} & \multirow{3}{*}{5.0} & $1.0-5.4$ & 1.92 \\
\hline 4 & 90 & 0.06 & 10 & 3.51 & & & \multirow{3}{*}{$3.4-5.5$} & \multirow{3}{*}{13.72} \\
\hline 5 & 90 & 0.06 & 10 & 4.38 & & & & \\
\hline 6 & 90 & 0.06 & 10 & 5.26 & \multirow{2}{*}{1.0} & \multirow{2}{*}{6.0} & & \\
\hline 7 & 90 & 0.06 & 10 & 6.14 & & & \multirow{3}{*}{$5.5-8$} & \multirow{3}{*}{23.11} \\
\hline 8 & 90 & 0.06 & 10 & 7.01 & \multirow{2}{*}{1.25} & \multirow{2}{*}{5.5} & & \\
\hline 9 & 90 & 0.06 & 10 & 7.89 & & & & \\
\hline 10 & 90 & 0.06 & 10 & 8.76 & \multirow{3}{*}{1.5} & \multirow{3}{*}{7.0} & \multirow{3}{*}{$8-10.8$} & \multirow{3}{*}{25.72} \\
\hline 11 & 90 & 0.06 & 10 & 9.64 & & & & \\
\hline 12 & 90 & 0.06 & 10 & 10.52 & & & & \\
\hline 13 & 90 & 0.06 & 10 & 11.39 & \multirow{3}{*}{2.5} & \multirow{3}{*}{8.0} & \multirow{3}{*}{$10.8-13.9$} & \multirow{3}{*}{20.03} \\
\hline 14 & 90 & 0.06 & 10 & 12.27 & & & & \\
\hline 15 & 90 & 0.06 & 10 & 13.15 & & & & \\
\hline 16 & 90 & 0.06 & 10 & 14.02 & 40 & \multirow{2}{*}{9.0} & \multirow{4}{*}{$13.9-17.2$} & \multirow{4}{*}{11.33} \\
\hline 17 & 90 & 0.06 & 10 & 14.90 & 4.0 & & & \\
\hline 18 & 90 & 0.06 & 10 & 15.78 & \multirow{2}{*}{4.5} & \multirow{6}{*}{10.0} & & \\
\hline 19 & 90 & 0.06 & 10 & 16.65 & & & & \\
\hline 20 & 90 & 0.06 & 10 & 17.53 & \multirow{4}{*}{5.0} & & \multirow{4}{*}{$17.2-20.8$} & \multirow{4}{*}{3.91} \\
\hline 21 & 90 & 0.06 & 10 & 18.41 & & & & \\
\hline 22 & 90 & 0.06 & 10 & 19.28 & & & & \\
\hline 23 & 90 & $8+8$ & 10 & 20.16 & & & & \\
\hline 24 & 90 & 0.06 & 10 & 21.04 & \multirow{2}{*}{7.0} & \multirow{2}{*}{12.0} & \multirow{2}{*}{$20.8-24.5$} & \multirow{2}{*}{0.26} \\
\hline 25 & 90 & 0.06 & 10 & 21.91 & & & & \\
\hline
\end{tabular}

For the wind files the Kaimal wind spectrum and IEC turbulent intensity model B was used throughout [27], along with a power law exponent of 0.14 and a surface roughness length of $0.03 \mathrm{~m}$. These turbulent wind velocity time histories were derived from 10 minute periodic stochastic turbulent mean winds, as recommended in the IEC 61400-3-1 standard [28]. The 10 minute interval is based on the wind variation spectral gap between the diurnal and turbulent peaks. However, analysis was conducted on 3 hour simulations, after the first 300 seconds was 
discarded to avoid transient responses. The choice of 3 hour simulation length was made partly due to the results presented by Li et al [29] where the relative error for 1 hour simulations exceeded $23 \%$ compared to 2 hour simulations. The other factor in this choice was that the statistical content of $1 \times 3$ hour simulation resembles that of $3 \times 1$ hour simulations, allowing the random seed requirement to be reduced [30]. This enabled the wind inflow to remain the same throughout a long simulation, but the floating turbine's response to vary, thus allowing adequate estimations of the fatigue of the tower to be obtained from the axial stress at different wind speeds, using a Rainflow cycle counting algorithm within MATLAB [31]. As the cross sectional area of the tower base is symmetrical, the axial stress can be found utilizing Equation 1 [29].

$$
\text { Axial Stress }=\left(\mathrm{F}_{\mathrm{zt}} / \mathrm{A}\right)+\left(\left(\mathrm{M}_{\mathrm{yt}} \cdot \operatorname{Cos}(\varnothing)-\mathrm{M}_{\mathrm{xt}} \cdot \operatorname{Sin}(\varnothing)\right)^{*}\left(\mathrm{r}_{\mathrm{o}} / \mathrm{I}\right)\right)
$$

where; $\mathrm{F}_{\mathrm{zt}}$ is the axial force at the base of the tower $(\mathrm{kN}), \mathrm{A}$ is the cross-sectional area of the tower base $\left(0.55 \mathrm{~m}^{2}\right)$, $M_{\mathrm{yt}}$ is the fore-aft moment at the base of the tower $(\mathrm{kN} / \mathrm{m}), \varnothing$ is the wind angle, $\mathrm{M}_{\mathrm{xt}}$ is the side to side moment at the base of the tower $(\mathrm{kN} / \mathrm{m}), \mathrm{r}_{\mathrm{o}}$ is the tower base cylinder central radius $(3.24 \mathrm{~m})$ and $\mathrm{I}\left(\right.$ as $\left.\mathrm{I}_{\mathrm{x}}=\mathrm{I}_{\mathrm{y}}\right)$ is the sectional moment of area $\left(\mathrm{m}^{4}\right)$.

Outputs are normalised with respect to the 'Feather Base Model' results, and are from the combined results from $3 \times 3$ hour simulations, each with 3 unique sets of random wind and wave seeds. The significant wave height and spectral peak period for each sea state are those specified in Table 2 at the relevant wind speed of a specific simulation run. All responses in turbulent wind conditions were obtained with all of the 16 wind turbine and 6 platform DOF enabled as detailed in depth by Jonkman et al [20].

\section{Results}

\subsection{Constant pitch angle below rated trends}

To enable the analysis of the effects of BTM and BTIP on tower axial fatigue life, an initial study on the effects on the constant pitch angle setting below rated was performed at mean turbulent winds of 8 and 13 mps. The responses at $18 \mathrm{mps}$ winds were unaffected as the wind speed did not drop below the rated speed of $11.4 \mathrm{mps}$, throughout the simulations. These were obtained with all the DOF relative to a 3 bladed HAWT enabled. Fig 2 shows the normalized ratio outputs as compared to the 'Feather Base Model' of a selection of load responses for a blade with a back twist angle of $-6^{\circ}$ as measured at the blade tip and applied at 3 initiation points along the blade length. The three initiation points presented are $75 \%, 87.5 \%$ and $92.75 \%$ as measured along the blade length from the root. Three different constant pitch angles below rated were analysed, $0^{\circ},-2^{\circ}$ and $-4^{\circ}$. 


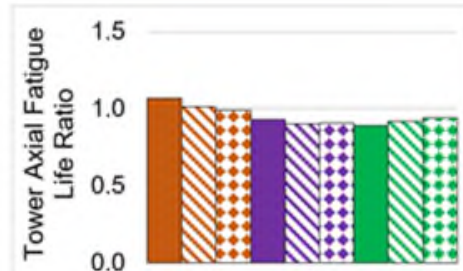

(a)

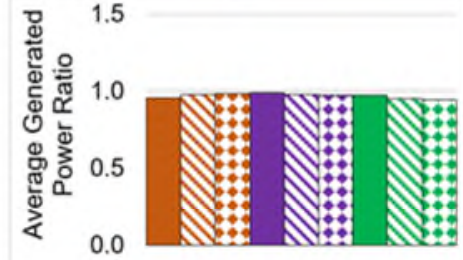

(c)

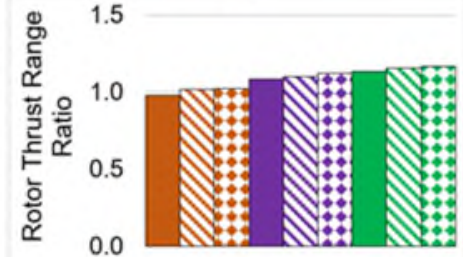

(e)

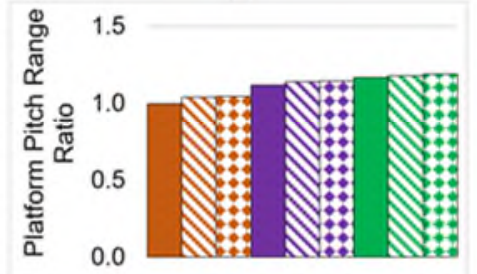

(g)

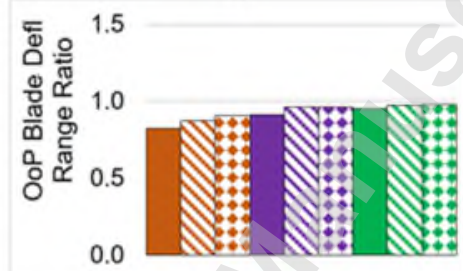

(i)

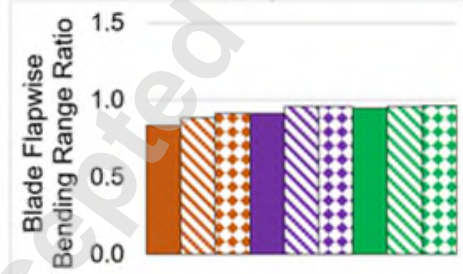

(k)

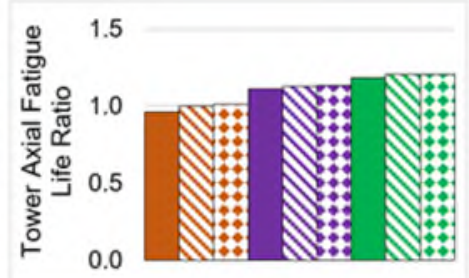

(b)

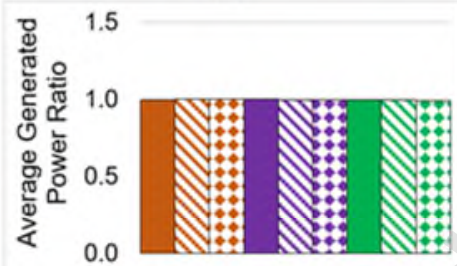

(d)

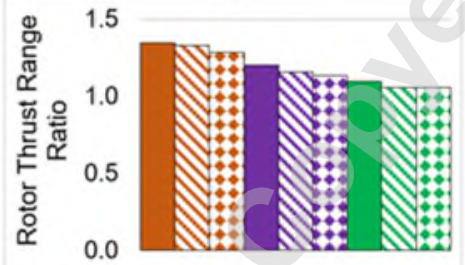

(f)

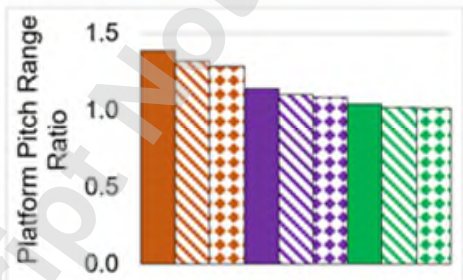

(h)

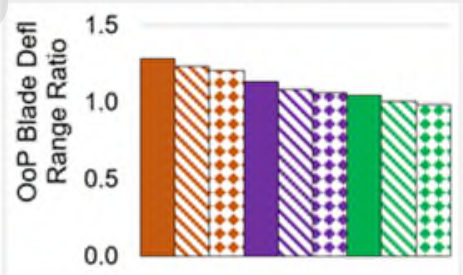

(j)

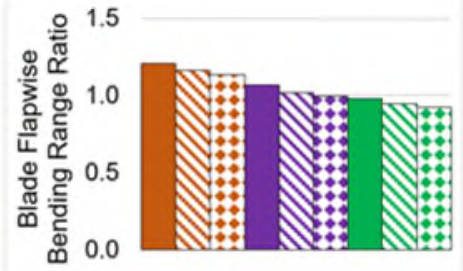

(I)

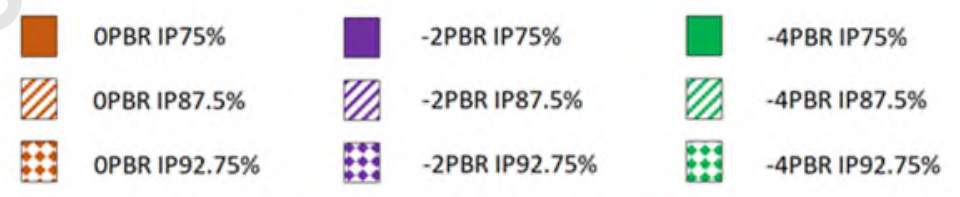

Figure 2. Normalized ratios to the 'Feather Base Model' of various outputs with increasing constant pitch angle below rated wind speed (PBR) and an increasing initiation point (IP), as measured from the blade root at $8 \mathrm{mps}$ (left) and $13 \mathrm{mps}$ (right) mean turbulent winds. 
When winds of $8 \mathrm{mps}$ were experienced, the tower axial fatigue life was highest when the constant pitch angle was set at $0^{\circ}$ and when the back twist initiation point started higher up the blade (Fig 2(a)). However the average generated power was only equal to the 'Feather Base Model' when the twist was either initiated close to the tip at a constant pitch angle of $0^{\circ}$ or when the angle was increased to $-2^{\circ}$ and closer to the root (Fig 2 (c). The rotor thrust, platform pitch, blade deflection and blade flapwise bending moment ranges decreased as the tower axial fatigue life increased, when the constant pitch angle was either $0^{\circ}$ or $-2^{\circ}$ (Fig $2(\mathrm{e}, \mathrm{g}, \mathrm{i}, \mathrm{k})$.

The tower axial fatigue life at $13 \mathrm{mps}$ steadily increased as the constant pitch angle increased from $0^{\circ}$ to $-4^{\circ}$, with the maximum seen at the initiation point of $92.75 \%$, hence the point closest to the tip (Fig 2(b)). However the generated energy was slightly reduced compared to that available from the 'Feather Base Model' at the higher fatigue life (Fig 2(d)). The rotor thrust, platform pitch, blade deflection and blade flapwise bending moment range responses all decreased with a corresponding increase in the tower axial fatigue life (Fig 2(f, h, j, 1). The decision was therefore made to have an intermediary constant pitch angle, such that the analysis stipulated a constant pitch angle setting of $0^{\circ}$ when the mean turbulent wind was $8 \mathrm{mps}$ and $-4^{\circ}$ when the mean was $13 \mathrm{mps}$.

\subsection{Back twist angle and initiation point trends}

Fig 3 shows the normalized ratio outputs as compared to the 'Feather Base Model', of a selection of relevant load analysis results at four different BTM and 3 different BTIPs along the blade radius (measured from the blade root). The tower fore-aft bending moment range was not included as this duplicated the trend of the tower axial fatigue life for all scenarios and hence was well represented by the fatigue life responses. The four different equispaced back twist angles were $-3^{\circ},-6^{\circ}-9^{\circ}$ and $-12^{\circ}$, as measured from the blade tip such that they all start to twist back towards feather at a specific initiation point. Three BTIP are presented at $75 \%, 87.5 \%$ and $92.75 \%$ as measured along the blade length from the root. A constant pitch angle below rated of $-4^{\circ}$ was also imposed at 13 mps as higher fatigue reductions were achieved with this setting. The original constant pitch angle of $0^{\circ}$ was applied when the mean winds conditions were lower at $8 \mathrm{mps}$. The constant pitch angle was not relevant when the higher $18 \mathrm{mps}$ winds were experienced as conditions remained within Region 3 throughout the simulations.

At mean turbulent winds of $8 \mathrm{mps}$, the tower axial fatigue life decreased as the back twist initiation point became closer to the blade tip at all back twist angles and increased as the back twist angle increased (Fig 3(a)). Therefore a the maximum fatigue life increase of over $12 \%$ compared to the 'Feather Base Model was realised at a back twist angle of $-12^{\circ}$ and an initiation point of $75 \%$. However, the trend clearly showed that increases in the tower axial fatigue life came at the expense of the generated power for all models, with an unacceptable drop in generated 
energy of $16 \%$ at the maximum fatigue life (Fig 3(a, d)). Decreases in the rotor thrust, platform pitch, blade deflection and blade flapwise bending moment ranges, all resulted in tower axial fatigue life increases (Fig 3(g, j, $\mathrm{m}, \mathrm{p})$ ).

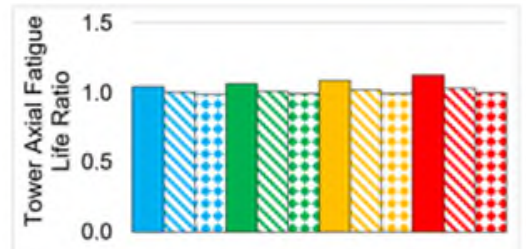

(a)

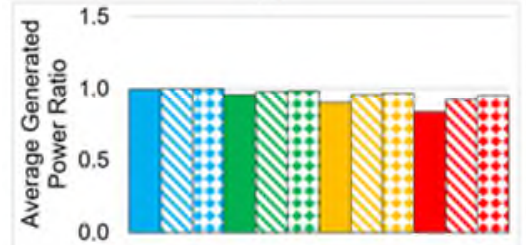

(d)

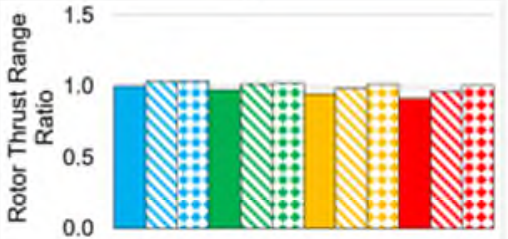

(g)

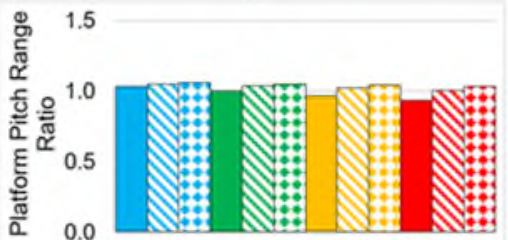

(j)

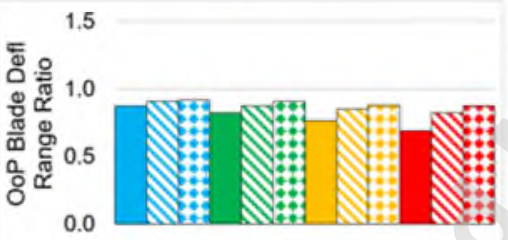

$(m)$

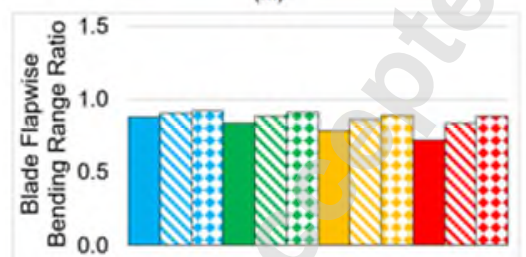

(p)

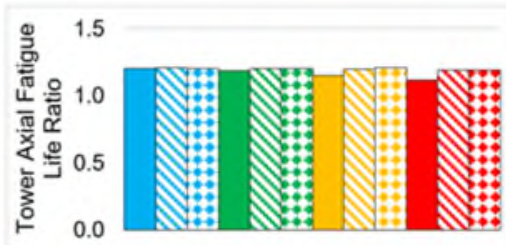

(b)

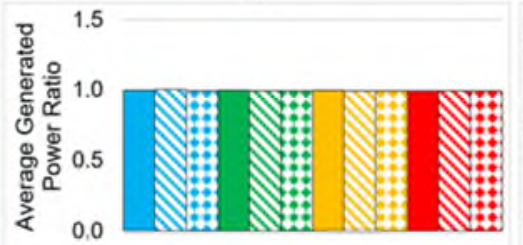

(e)

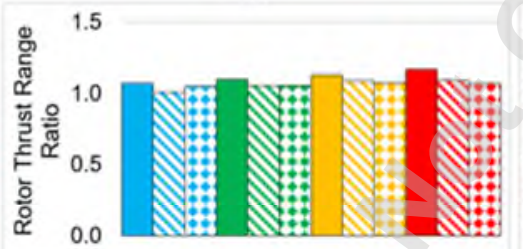

(h)

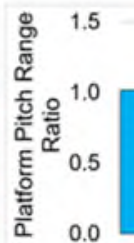

1.5

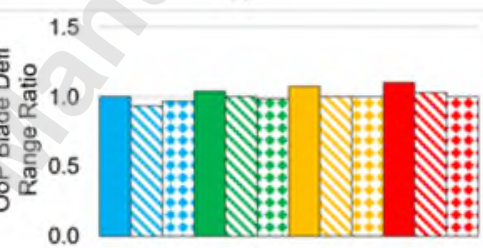

(n)

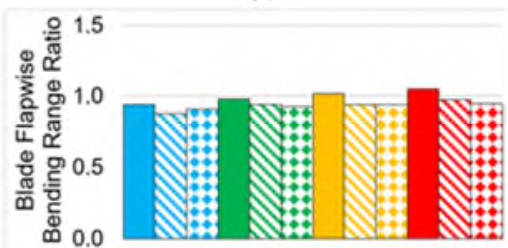

(q)

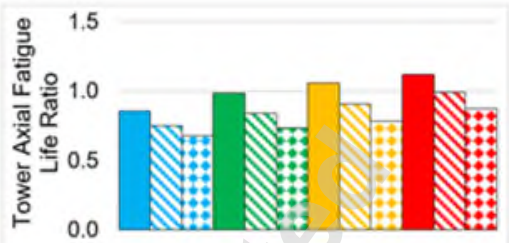

(c)

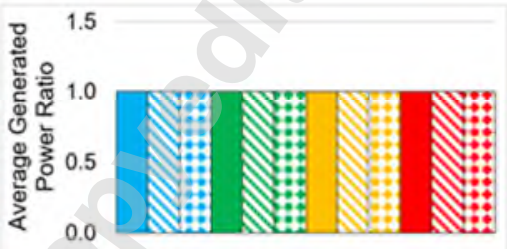

(f)

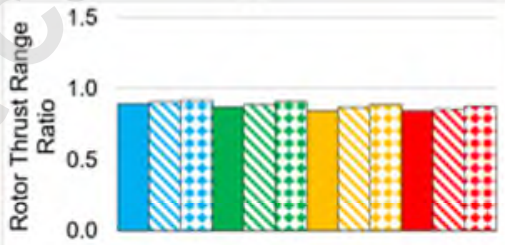

(i)

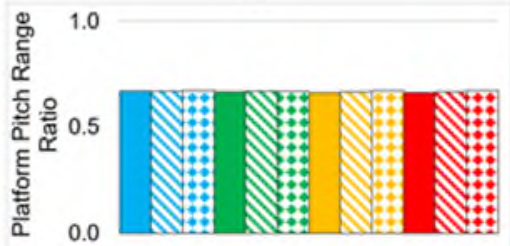

(I)

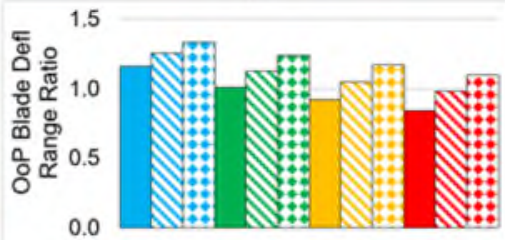

(o)

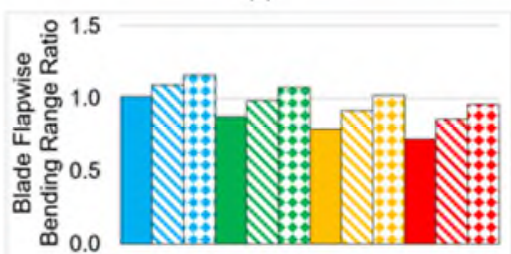

(r)

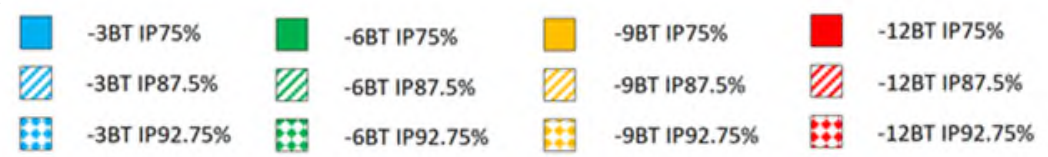

Figure 3. Normalized ratios to the 'Feather Base Model' of various outputs with increasing back twist angles (BT) and an increasing initiation point (IP), as measured from the blade root at $8 \mathrm{mps}$ (left), $13 \mathrm{mps}$ (centre) and 18 mps (right) mean turbulent winds. 
The tower axial fatigue life at $13 \mathrm{mps}$ mean turbulent winds achieved an increase $+20 \%$ compared to the 'Feather Base Model' for all back twist angles initiated at $92.75 \%$ from the blade root, hence the initiation point closest to the tip (Fig 3(b)). The back twist angle had little influence except for a notable drop for both $-9^{\circ}$ and $-12^{\circ}$ at the initiation point of $75 \%$, the point closest to the blade root. A slight drop of less than $1 \%$ in generated energy was also seen (Fig 3(e)).

The rotor thrust, platform pitch, blade deflection and blade flapwise bending moment range responses followed the same trend as at $8 \mathrm{mps}$, such that a decrease in all these ranges generally corresponded with an increase in the tower axial fatigue life (Fig 3(h, k, n, q)).

At $18 \mathrm{mps}$ the variation in tower axial fatigue life due to blade twist angle and initiation point was more pronounced such that the fatigue life increased by $12 \%$ compared to the 'Feather Base Model' as the BTM increased to $12^{\circ}$ but reduced as the BTIP approached the tip(Fig 3(c)). Generated energy was unaffected by both back twist angle and initiation point (Fig 3(f)). Decreases in the rotor thrust, blade deflection and blade flapwise bending moment ranges had corresponding increases in tower axial fatigue life (Fig 3(i, o, r), however the platform pitch response range varied little, although the fatigue life variation between models was up to $44 \%$ in range.

In order to take into account the likely occurrence of the different wind speed cases, a weighted analysis that takes account of the distribution of probability of occurrence was conducted. This was applied to the tower axial fatigue life and average generated power for each model, corresponding to the ' $\%$ of total operating time' seen in Table 2 within the Section 3, for each of the 3 mean turbulent wind speeds analysed. These results were then compared against the weighted 'Feather Base Model' results and are presented in Table 3.

Table 3. Weighted tower axial fatigue life and generated power for each back twist model.

\begin{tabular}{|c|c|c|c|c|c|c|c|c|c|c|c|c|}
\hline $\begin{array}{l}\text { Test ID for VSVP-S 'Stall Back } \\
\text { Twist Models' }\end{array}$ & BT 1 & BT 2 & BT 3 & BT 4 & BT 5 & BT 6 & BT 7 & BT 8 & BT 9 & BT 10 & BT 11 & BT 12 \\
\hline $\begin{array}{l}\text { Initiation point along the blade, } \\
\text { measured from the root }(\%)\end{array}$ & 75.00 & 87.50 & 92.75 & 75.00 & 87.50 & 92.75 & 75.00 & 87.50 & 92.75 & 75.00 & 87.50 & 92.75 \\
\hline Back twist angle at the tip $\left(^{\circ}\right)$ & -3 & -3 & -3 & -6 & -6 & -6 & -9 & -9 & -9 & -12 & -12 & -12 \\
\hline $\begin{array}{l}\text { Tower axial fatigue life change from } \\
\text { 'Feather Base Model' (\%) } \\
(+ \text { ve means a longer tower life) }\end{array}$ & 8.29 & 5.10 & 3.14 & 10.22 & 6.50 & 4.11 & 10.98 & 7.53 & 4.94 & 12.09 & 8.85 & 5.91 \\
\hline $\begin{array}{l}\text { Average generated power change } \\
\text { from 'Feather Base Model' (\%) }\end{array}$ & -0.47 & -0.14 & -0.55 & -1.14 & -0.81 & -0.80 & -2.36 & -1.36 & -1.16 & -3.88 & -2.03 & -1.58 \\
\hline
\end{tabular}

For each back twist model an overall increase in fatigue life was seen, ranging from approximately $3 \%$ to $12 \%$.

The higher fatigue life increases were seen when the twist was initiate closer to the root at a distance of $75 \%$ along 
the blade and with higher back twist angles. However, increases in fatigue life was at the expense of the generated power, with a maximum loss from the combined results over all three wind speeds of $3.88 \%$.

\section{Discussion}

From the analysis of the effects on the tower axial fatigue life of varying the constant pitch angle setting (when the winds are below the rated wind speed of $11.4 \mathrm{mps}$ ), it was clearly highlighted that reductions in the variance of both blade flapwise bending moment and blade deflection created a corresponding reduction in the range of the rotor thrust (Fig 2). The reduced thrust force range then reduced the range of both the platform pitch and tower fore-aft motion, which consequently increased the tower axial fatigue life. Although the range of the thrust force is lower, there is a significant rise in the thrust force from rated wind speed to the cut-out wind speed of $25 \mathrm{~m} / \mathrm{s}$. This could cause the mean inclination of the floating turbine to increase. To account for this a semi-submersible floating turbine can incorporate an active water ballast system that can be adjusted as required during the different environmental operating conditions.

The responses in mean winds of 8 and $13 \mathrm{mps}$ winds to the constant pitch angle changes were opposed to each other. Hence, the rotor thrust range decreased as the constant pitch angle became smaller and the angle of attack induced at $8 \mathrm{mps}$ was reduced, but the opposite response was observed when operating in $13 \mathrm{mps}$ such that increasing the angle of attack at the higher wind speeds reduced the thrust range and hence increased the tower axial fatigue life.

At both 8 and 18 mps mean turbulent winds, the tower axial fatigue life decreased as the back twist initiation point became closer to the blade tip at all back twist angles and increased as the back twist angle increased (Fig $3(\mathrm{a}, \mathrm{c}))$. Thus the aerodynamic damping and hence thrust reduction provided by the back twist angle was at its highest for an angle of $-12^{\circ}$ initiated at the point closest to the blade root for both environments. However, the damping provided by this blade profile is lower at $18 \mathrm{mps}$ than at $8 \mathrm{mps}$ when compared to the 'Feather Base Model' responses at these wind speeds. Also, although the tower axial fatigue life increase with this back twist blade profile is $12 \%$ higher at $8 \mathrm{mps}$ than for pitch-to-feather control, this comes at an unacceptable loss of $16 \%$ in the average energy generated. Thus the chosen back twist profile must find a compromise between tower fatigue life increase and energy generation.

For all blade profiles a large increase in fatigue life was seen for all the pitch-to-stall back twist models at $13 \mathrm{mps}$ winds, which exceeded $20 \%$ for all back twist angles initiated at 92.75\%, compared to the 'Feather Base Model'

(Fig 3 (b)). There was no noticeable trend however, as changes to the back twist angle and initiation point had 
much less influence at $13 \mathrm{mps}$, except for a notable drop in fatigue life for the higher back twist angles when the initiation point was closest to the blade root. This drop corresponds to an increase in the platform pitch, blade deflection and blade flapwise bending moment response ranges, however, the same increases were seen at lower back twist magnitudes nearer the root without causing a fatigue life reduction. A slight drop (of up to $1 \%$ ) in generated energy was also seen for all back twist models at this wind.

The rotor thrust, blade deflection and blade flapwise bending moment range responses followed the same trends, such that if their response to variations in the wind speed was reduced, a corresponding increase was seen in the tower fatigue life as the tower motion was also reduced.

Reductions in the platform pitch response at 8 and 13mps also created an increase in the tower axial fatigue life. However, at $18 \mathrm{mps}$ the platform pitch response was unaffected by rotor thrust reductions, even though these reductions directly translated into reduced tower fore-aft motion and hence increased tower axial fatigue life that varied by up to $44 \%$ in range. This therefore concurs with the findings of Lackner [11], where tower load reductions are not always achieved through decreases in the platform pitch response, and hence tower motion reduction, that impedes the inertial and gravitational loads, should be the target.

A weighted analysis of all the back twist profile results allowed a compromise between tower fatigue life increase and energy generation reduction to be considered. From that weighted analysis an optimum back twist profile for the presented semi-submersible FOWT in the defined environment conditions can be identified. When specifying maximum energy generation, then a back twist angle of $-3^{\circ}$ initiated at $87.5 \%$ along the blade from the root is optimum, with a tower axial fatigue life increase compared to the 'Feather Base Model' of 5.1\%, achieved with just a $0.14 \%$ reduction in energy. However, if an energy loss of up to $1 \%$ was acceptable then although this would stipulate that the back twist angle did not exceed $-6^{\circ}$, suggesting an optimum initiation point would be more complex as a defined trend does not appear within the weighted results.

\section{Conclusions}

Within most numerical studies, the turbines mounted on floating platforms are generally those that have been designed for fixed bases, however the induced tower base bending moments of a FOWT have been shown to increase by up to 1.6 times. If these loads are not reduced, then towers must be strengthened which results in increased costs and weight for both the tower and floating platform. The regulation of the power generation is also seen to suffer for a FOWT, due to the need to avoid the negative damping created by the drop in rotor thrust of a turbine with pitch-to-feather control. 
The aim of this research was to investigate the effects on the tower axial fatigue life of both increasing the back twist angle and the point along the blade where the back twist initiates at, for a VSVP-S controlled 5MW HAWT. This was coupled to a semi-submersible floating platform with a constant pitch angle setting below rating. Then, through examination of the aerodynamic damping, and hence changes to the rotor thrust created by the different blade profiles, the trends were identified when the FOWT was operating in three different mean turbulent wind environments. This then allowed a further weighted analysis accounting for wind frequency at the specific site, so that an optimum blade profile for minimum energy loss could be presented. Also, by utilizing VSVP-S control, the issues relating to the negative damping phenomenon were avoided.

The proposed approach has achieved its aim by highlighting the trends at each mean wind speed analysed, due to the introduction of a back twist towards feather along part of the blade length, which then allowed an optimum blade profile for tower fatigue reduction, with minimum energy loss to be presented from combined weighted responses.

The rotor thrust range was seen to decrease when the constant pitch angle decreased, and hence the angle of attack decreased at $8 \mathrm{mps}$ winds, which resulted in a reduction in the fore-aft motion of the tower. Hence, the optimum constant pitch angle of $0^{\circ}$ was the most appropriate at this wind speed. For $13 \mathrm{mps}$ winds, the trend from the effects of a constant pitch angle were the complete opposite, such that increases in the angle of attack reduced the thrust range and hence increased the tower axial fatigue life. Therefore, the optimum constant pitch angle of $-4^{\circ}$ was the most applicable for this environment. Two constant pitch angles were therefore imposed, that came into effect when the turbine was operating in winds below rated, such that at wind speed between 8 mps and 13 mps, the constant pitch angle setting would increase from $0^{\circ}$ to $-4^{\circ}$.

The trends for both increases in the back twist angle and in the initiation point distance along the blade were the same in both 8 and 18 mps winds, such that the tower axial fatigue life was seen to decrease as the back twist initiation point moved closer to the blade tip (for all of the back twist angles), and increased as the back twist angle increased.

There were no noticeable trends seen due to changes in either the back twist angle or initiation point at $13 \mathrm{mps}$. However, the tower fatigue life was seen to exceed that achieved by the 'Feather Base Model' for all the back twist models at 13 mps winds, with a $20 \%$ increase achieved for all back twist angles initiated at $92.75 \%$ along the blade length. 
At $18 \mathrm{mps}$ the platform pitch response was unaffected by rotor thrust reductions, which agrees with the findings of Lackner [11] such that decreased tower loads do not always correspond to reductions in the platform pitch response range.

A weighted analysis, where maximum energy generation was chosen as the overriding constraint, identified the optimum blade profile, for this FOWT in this specific environment, to have a back twist angle of $-3^{\circ}$ that initiated at a point $87.5 \%$ along the blade as measured from the root. This is identified as 'Stall Back Twist Model 5' in Table 1 of Section 2.3. This provided a tower axial fatigue life increase compared to the 'Feather Base Model' of $5.1 \%$, achieved with just a $0.14 \%$ reduction in energy.

The effect of a higher constant pitch angle below rated for operation in mean winds around 13 mps could identify even higher reductions in tower fatigue. This knowledge could also be particularly applicable for turbine life increase, as the decommissioning date approaches, such that the constant pitch angle below rated of a VSVP-S blade could be increased to extend the life of a turbine tower, a situation where the energy loss may be deemed to be worth the increased operational life achieved.

The blade profiles presented in this study give an initial indication of the trends and possible benefits for turbine towers mounted on floating platforms that utilize a VSVP-S and back twisting blades. However, the blade designs are preliminary, and a fully detailed design that could provide a smoother transition in the thrust-speed curve could be beneficial, as would more detailed analyse of the loads experienced by the rotor and all of the other turbine systems, by the introduction of a back twist to the blade. A future study would also need to examine the effects on the tower fatigue life from shear stress due to torsion. Further analysis on acceptable energy loss for tower fatigue life increase would also be beneficial in selecting an optimum back twist blade profile for a specific site.

Funding: This work was funded by the United Kingdom Engineering and Physical Sciences Research Council (EPSRC), grant EP/L016303/1, for Doctoral Training in Renewable Energy Marine Structures (REMS).

Conflicts of Interest: The authors declare that they have no conflict of interest.

\section{References}

[1] Knutson, T.R., Kam, J., Zeng, F. and Wittenberg, A.T., 2018. CMIP5 model-based assessment of anthropogenic influence on record global warmth during 2016. Bulletin of the American Meteorological Society, 99(1), pp.S11-S15. 
[2] First, P., 2018. Global warming of 1.5 C An IPCC Special Report on the impacts of global warming of $1.5 \mathrm{C}$ above pre-industrial levels and related global greenhouse gas emission pathways, in the context of strengthening the global response to the threat of climate change, sustainable development, and efforts to eradicate poverty.

[3] Europe, W., 2018. Wind energy in Europe in 2018, trends and statistics. WindEurope, Brussels, Belgium

[4] Gwec, 2017. Global Wind Report: Annual market update 2017. URL https://gwec.net/publications/ globalwind-report-2/.[Accessed Sept 22, 2019].

[5] Robertson, A. and Jonkman, J., 2011. Loads analysis of several offshore floating wind turbine concepts. In The Twenty-first International Offshore and Polar Engineering Conference. International Society of Offshore and Polar Engineers.

[6] Larsen, T. and Hanson, T., 2007. A method to avoid negative damped low frequent tower vibrations for a floating, pitch controlled wind turbine. In Journal of Physics: Conference Series (Vol. 75, No. 1, p. 012073). IOP Publishing.

[7] Ward, D., Collu, M. and Sumner, J., 2019. Reducing Tower Fatigue through Blade Back Twist and Active Pitch-to-Stall Control Strategy for a Semi-Submersible Floating Offshore Wind Turbine. Energies, 12(10), p.1897.

[8] Macquart, T. and Maheri, A., 2019. A stall-regulated wind turbine design to reduce fatigue. Renewable energy, 133, pp.964-970.

[9] Bossanyi, E., 2003. Wind turbine control for load reduction. Wind Energy: An International Journal for Progress and Applications in Wind Power Conversion Technology, 6(3), pp.229-244.

[10] Jonkman, J., 2007. Dynamics modeling and loads analysis of an offshore floating wind turbine. National Renewable Energy Laboratory, Golden, CO, Technical Report No. NREL/TP-500-41958.

[11] Lackner, M.A., 2013. An investigation of variable power collective pitch control for load mitigation of floating offshore wind turbines. Wind Energy, 16(4), pp.519-528.

[12] Fleming, P., Peiffer, A. and Schlipf, D., 2019. Wind Turbine Controller to Mitigate Structural Loads on a Floating Wind Turbine Platform. Journal of Offshore Mechanics and Arctic Engineering, 141(6), p.061901.

[13] Stäblein, A., Hansen, M. and Verelst, D., 2017. Modal properties and stability of bend-twist coupled wind turbine blades. Wind Energy Science, 2, pp.343-360. 
[14] Merz, K., 2011. Conceptual design of a stall-regulated rotor for a deepwater offshore wind turbine. Norwegian University of Science and Technology

[15] Méndez, J. and Greiner, D., 2006, September. Wind blade chord and twist angle optimization by using genetic algorithms. In Proceedings of the Fifth International Conference on Engineering Computational Technology, B. Topping, G. Montero, and R. Montenegro, eds., Civil-Comp Press, Las Palmas de Gran Canaria, Spain, Sept (Vol. 6, pp. 12-15).

[16] Ostachowicz, W., McGugan, M., Schröder-Hinrichs, J. and Luczak, M. eds., 2016. MARE-WINT: new materials and reliability in offshore wind turbine technology. Springer.

[17] Lobitz, D. and Veers, P., 2003. Load mitigation with bending/twist-coupled blades on rotors using modern control strategies. Wind Energy: An International Journal for Progress and Applications in Wind Power Conversion Technology, 6(2), pp.105-117.

[18] Robertson, A., Jonkman, J., Masciola, M., Song, H., Goupee, A., Coulling, A. and Luan, C., 2014. Definition of the semisubmersible floating system for phase II of OC4. National Renewable Energy Laboratory, Golden, CO, Technical Report No. NREL/TP-5000-60601

[19] Robertson, A., Jonkman, J., Vorpahl, F., Popko, W., Qvist, J., Froyd, L., Chen, X., Azcona, J., Uzungoglu, E., Guedes Soares, C. and Luan, C., 2014. Offshore code comparison collaboration, continuation within IEA Wind task 30: phase II results regarding a floating semisubmersible wind system. National Renewable Energy Laboratory, Golden, CO, Technical Report No. NREL/CP-5000-61154

[20] Jonkman, J., Butterfield, S., Musial, W. and Scott, G., 2009. Definition of a 5-MW Reference Wind Turbine for Offshore System Development. National Renewable Energy Laboratory, Golden, CO, Technical Report No. NREL/TP-500-38060

[21] Jonkman, J. and Buhl Jr, M., 2005. FAST user's guide. National Renewable Energy Laboratory, Golden, CO, Technical Report No. NREL/EL-500-38230.

[22] Goupee, A., Fowler, M., Kimball, R., Helder, J. and de Ridder, E., 2014. Additional wind/wave basin testing of the DeepCwind semi-submersible with a performance-matched wind turbine. In ASME 2014 33rd International Conference on Ocean, Offshore and Arctic Engineering. American Society of Mechanical Engineers Digital Collection.

[23] Namik, H. and Stol, K., 2010. Individual blade pitch control of floating offshore wind turbines. Wind Energy: An International Journal for Progress and Applications in Wind Power Conversion Technology, 13(1), pp.74-85. 
[24] Wright, A. and Fingersh, L., 2008. Advanced control design for wind turbines. Part I: Control Design, Implementation, and Initial Tests. National Renewable Energy Laboratory, Golden, CO, Technical Report No. NREL/EL-500-42437

[25] Hansen, M., Hansen, A., Larsen, T., Øye, S., Sørensen, P. and Fuglsang, P., 2005. Control design for a pitchregulated, variable speed wind turbine.

[26] Geos, F., 2001. Wind and wave frequency distributions for sites around the British Isles. Great Britain, Health and Safety Executive.

[27] EN, B., 2006. 61400-1: 2005+ A1: 2010: Wind Turbines-Design Requirements. British Standards Institution: London, UK.

[28] EN, B., 2017. 61400-3-1: Design requirements for offshore wind turbines. British Standards Institution: London, UK.

[29] Li, H., Hu, Z., Wang, J. and Meng, X., 2018. Short-term fatigue analysis for tower base of a spar-type wind turbine under stochastic wind-wave loads. International Journal of Naval Architecture and Ocean Engineering, 10(1), pp.9-20.

[30] Kvittem, M., and Moan, T., 2015. Time domain analysis procedures for fatigue assessment of a semisubmersible wind turbine. Marine Structures, 40, pp.38-59.

[31] Niesłony, A., 2009. Determination of fragments of multiaxial service loading strongly influencing the fatigue of machine components. Mechanical Systems and Signal Processing, 23(8), pp.2712-2721. 


\section{Table caption list}

Table 1. Model parameters

Table 2. Average annual environmental conditions at grid point $59.300 \mathrm{~N} 0.475 \mathrm{E}$

Table 3. Weighted tower axial fatigue life and generated power for each back twist model.

\section{Figure caption list}

Figure 1. Pitch-to-stall feedback proportional-integral controller

Figure 2. Normalized ratios to the 'Feather Base Model' of various outputs with increasing constant pitch angle below rated wind speed (PBR) and an increasing initiation point (IP), as measured from the blade root at $8 \mathrm{mps}$ (left) and 13 mps (right) mean turbulent winds.

Figure 3. Normalized ratios to the 'Feather Base Model' of various outputs with increasing back twist angles (BT) and an increasing initiation point (IP), as measured from the blade root at $8 \mathrm{mps}$ (left), $13 \mathrm{mps}$ (centre) and 18 mps (right) mean turbulent winds. 